\title{
Dual-Band Balanced Bandpass Filter with Common-Mode Suppression Based on Electrically Small Planar Resonators
}

\author{
Paris Velez, Member IEEE, Jordi Bonache, Member IEEE, and Ferran Martín, Fellow IEEE
}

\begin{abstract}
The design of fully planar dual-band balanced bandpass filters with common-mode noise suppression is reported. The proposed filters are based on electrically small resonators coupled through admittance inverters. For design purposes, the circuit models of the considered resonators are reported. The key aspect for selective mode suppression (i.e., common-mode rejection in the differential-mode pass bands) is related to symmetry properties. Thus, for the differential-mode the symmetry plane is an electric wall, and the equivalent circuit for that mode provides dual-band functionality. Conversely, for the common-mode the symmetry plane is a magnetic wall, and the equivalent circuit exhibits a rejection band. As a proof of concept, the design of an order-2 Chebyshev dual-band balanced bandpass filter with center frequencies $f_{1}=1.8 \mathrm{GHz}$ (GSM band) and $f_{2}=2.4 \mathrm{GHz}$ (Wi-Fi band), fractional bandwidth $F B W=7 \%$, and ripple level $L_{A r}=0.01 \mathrm{~dB}$ is reported.
\end{abstract}

Index Terms-Balanced filters, common-mode suppression, dual-band filters, split ring resonators, semi-lumped elements.

\section{INTRODUCTION}

$\mathrm{I}$ $\mathrm{N}$ recent years, the use of balanced (differential-mode) circuits and systems (e.g., in high speed digital circuits) has increased due to their inherent high immunity to noise, electromagnetic interference (EMI) and crosstalk, as compared to the single-ended counterparts. The miniaturization of microwave components is also one of the most important challenges in communications systems. Within this framework, this work is focused on the design of dual-band balanced bandpass filters with compact size, high differentialmode selectivity, and efficient common-mode rejection. One possibility is to cascade two baluns at the input and output ports of a dual-band structure based on coupled stepped impedance resonators (SIRs) [1], but this approach increases device size (a SIR-based differential-mode dual-band bandpass filter without baluns is reported in [2]). In [3], the authors use an approach based on coupled half-wavelength SIR resonators, resulting in small size but limited commonmode rejection. In [3],[4] common-mode suppression is enhanced at the expense of using loaded lumped elements. More recently, in [5] the authors reported an approach based on asymmetrical coupled lines. In these balanced filters, the

This work was supported by MINECO-Spain (projects TEC2013-40600R), Generalitat de Catalunya (project 2014SGR-157), ICREA (who awarded Ferran Martín), and by FEDER funds.

P. Vélez, J. Bonache, and F. Martín are with GEMMA/CIMITEC (Departament d'Enginyeria Electrònica), Universitat Autònoma de Barcelona. 08193 BELLATERRA (Barcelona). Spain. E-mail: Ferran.Martin@uab.es. final size is not compact due to the use of distributed resonators. In this work, we propose the design of a fully planar and compact dual-band balanced bandpass filter with efficient and intrinsic common-mode rejection, based on electrically small resonators. The design is based on the lumped element circuit models of the considered resonators.

\section{DUAL-BAND BANDPASS FILTERS}

It is possible to design a multi-band bandpass filter with the circuit model of Fig.1. However, in order to synthesize a standard response (e.g. Chebyshev), only dual-band functionality can be obtained, due to the need to set the equalripple and fractional bandwidth $(F B W)$ at each band. Dualband functionality can be obtained in a two-step process, where first a lowpass to bandpass transformation is used [6]

$$
\Omega=\frac{\Omega_{c}}{F B W_{0}}\left(\frac{\omega^{\prime}}{\omega_{0}}-\frac{\omega_{0}}{\omega^{\prime}}\right)
$$

$\Omega$ and $\omega$ ' being the angular frequency of the low-pass and band-pass filter, respectively. $F B W_{0}$ and $\omega_{0}$ are the fractional bandwidth and central frequency of the band-pass filter and $\Omega_{\mathrm{c}}$ is the angular cut-off frequency of the low-pass filter. Then, to obtain dual-band behavior, a second transformation is applied to the single-band band-pass filter [6]

$$
\omega^{\prime}=\frac{\omega_{0}}{F B W_{0}}\left(\frac{\omega}{\omega_{0}}-\frac{\omega_{0}}{\omega}\right) F B W_{0}=\left(\frac{\omega_{2}-\omega_{1}}{\omega_{0}}\right) \omega_{0}=\sqrt{\omega_{2} \omega_{1}}
$$

where $\omega$ is the angular frequency of the dual-band bandpass filter, and $\omega_{1}$ and $\omega_{2}$ are the angular central frequencies of the first and second band, respectively. If these transformations are applied, the lumped element equivalent circuit of Fig.1, with identical $F B W$ for each band, is derived (the narrow band approximation, with $F B W_{D B}$ typically less than $10 \%$ [7], is necessary). In this case, the $F B W_{D B}$ can be calculated by

$$
F B W_{D B}=F B W_{1}=F B W_{2}=F B W_{0}\left(\frac{\omega_{2}-\omega_{1}}{\omega_{2}+\omega_{1}}\right)
$$

Due to the difficulty of implementing a dual-band bandpass filters with the configuration of Fig.1 (series and shunt branches include both series and parallel LC resonators) in microstrip technology, and to the fact that the $F B W$ of each band is set to less than $10 \%$, we have considered the alternative network of Fig. 2, where the series branch is replaced by admittance inverters ( $J$-inverters).

In this scenario, the element values of the circuit of Fig. 2, composed by shunt series/parallel resonators and admittance inverters, implemented by means of $90^{\circ}$ (at $\left.\omega_{0}^{2}=\omega_{1} \omega_{2}\right)$ transmission line sections are obtained from a set of equations 
that depend on the low-pass filter prototype coefficients $\left(g_{i}\right)$ and filter specifications, such as $F B W$ and central angular frequencies $\left(\omega_{1}\right.$ and $\left.\omega_{2}\right)$ [7]:

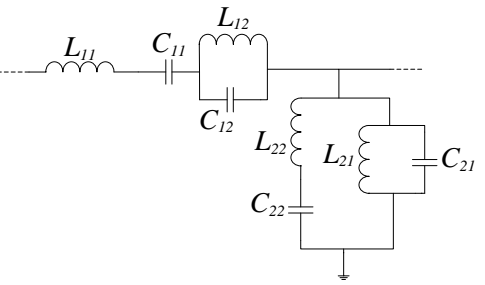

Fig.1. Circuit model of the 2-pole dual-band bandpass filter.

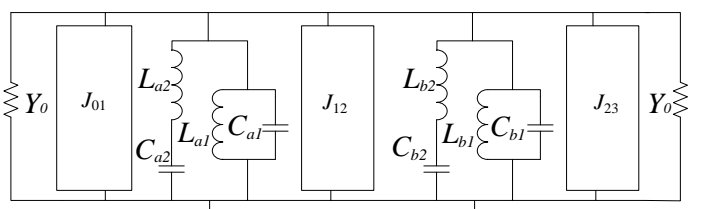

Fig.2. Network a 2-pole Chebyshev dual-band bandpass filter with shunt resonators and admittance inverters.

$$
\begin{gathered}
L_{a i}=\frac{g_{0} Y_{0}}{J_{01}^{2}} C_{1 i}, \quad L_{b i}=\frac{J_{01}^{2}}{g_{0} Y_{0} J_{12}^{2}} L_{2 i}, \quad i=1,2 \\
L_{a 1} C_{a 1}=L_{a 2} C_{a 2}=L_{b 1} C_{b 1}=L_{b 2} C_{b 2}=\frac{1}{\omega_{0}^{2}} \\
J_{12}=\frac{J_{01} J_{23}}{Y_{0}} \sqrt{\frac{g_{3}}{g_{0}}}
\end{gathered}
$$

where the parameters $C_{1 i}$ and $L_{2 i}$ are related to the canonical 2pole Chebyshev dual-band bandpass filters based on the circuit of Fig.1.

\section{TOPOlOgy AND Circuit Models Of THE Resonators}

To implement the dual-band balanced filter according to the differential-mode version of the network of Fig. 2, mirrored open complementary split ring resonators (OCSRRs), see Fig. 3 , are used for the LC parallel resonators $\left(L_{a l}-C_{a l}\right.$ and $\left.L_{b l}-C_{b l}\right)$, whereas the for the $\mathrm{LC}$ series shunt resonators $\left(L_{a 2}-C_{a 2}\right.$ and $\left.L_{b 2}-C_{b 2}\right)$, interdigital capacitors and meander inductors (connected to ground by vias) are considered. The circuit model of the mirrored OCSRRs, placed between the pair of lines as shown in Fig. 3(a), is depicted in Fig. 3(b) [8]. For the differential mode, $C_{2}$ is grounded, and the equivalent circuit is described by three elements: $L, C=C_{a l}=C_{b l}=C_{p}+C_{1}$ and $L_{p}=$ $L_{a l}=L_{b l}$. Under common-mode excitation, the symmetry plane is a magnetic wall, and the equivalent circuit shows a rejection behavior useful to suppress the common-mode. As an added value, the mirrored OCSRRs introduce a transmission zero for the differential mode, above the second differential-mode pass band, which is useful to improve filter selectivity. The frequency of this transmission zero can be calculated [in reference to the model of Fig. 3(b)] as

$$
f_{\mathrm{Z}}^{d d}=\frac{1}{2 \pi} \sqrt{\frac{1}{C}\left(\frac{1}{L}+\frac{1}{L_{p}}\right)}
$$

Fig. (4) shows the electromagnetic simulation (using Agilent Momentum) for the topology of Fig. 3(a), compared to the response of the equivalent circuit of Fig. 3(b) for both modes (differential and common). The response of the ideal LC parallel resonator is also included. The element values of the equivalent circuit for the differential OCSRR are derived from the susceptance slope at the reflection zero frequency (Fig. 4(c), the reflection zero frequency, and the transmission zero (that must be set to a certain value) for the differential mode. The remaining circuit element, $C_{2}$, is determined from the position of the transmission zero for the common mode $\left(C_{2}\right.$ is controlled by means of the metallic part around the OCSRR). The final topology is determined by curve fitting. It can be seen the good agreement between the electromagnetic and circuit responses (except, obviously, for the differential-mode transmission zero, which is absent in the ideal LC response).

(a)
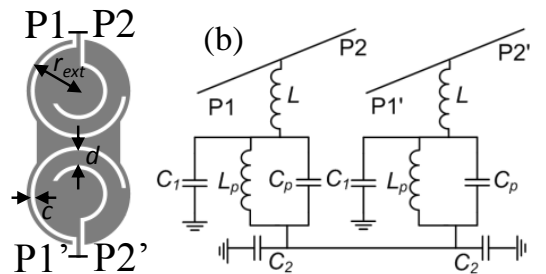

Fig. 3. Typical topology of a differential microstrip line section loaded with a pair of mirrored OCSRRs (a) and lumped element equivalent circuit model (b) [9]. Dimensions are (in mm): $r_{e x t}=1.9, c=0.16$ and $d=0.65$.
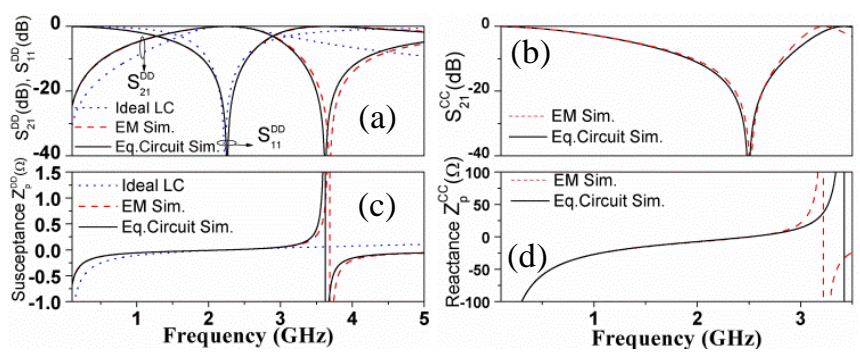

Fig. 4.S-parameters (a-b) and susceptance-reactance (c-d) of the layout of Fig.3, compared to the equivalent circuit and the ideal $\mathrm{LC}$ parallel resonator. The element values of the equivalent circuit are: $L=0.89 \mathrm{nH}, C=C_{p}+C_{l}=$ $3.36 \mathrm{pF}, L_{p}=1.481 \mathrm{nHand} C_{2}=2.1 \mathrm{pF}$. The considered substrate for the EM simulations is RogersRO3010 with dielectric constant $\varepsilon_{\mathrm{r}}=10.2$ and thickness $h=0.254 \mathrm{~mm}$. The unloaded quality factor of the OCSRR is $Q_{u}\left(\omega_{0}\right)=85$.

For the LC shunt-connected series resonators $\left(L_{a 2}-C_{a 2}\right.$ and $L_{b 2^{-}}$ $C_{b 2}$ ), in order to maximize the series inductance, a window in the ground plane was opened. The proposed equivalent circuit of the shunt-connected series resonator is shown in Fig. 5(b). The procedure to determine the layout is similar to the case of the OCSRRs, i.e., parameter extraction and curve fitting. However, the position of the reflection frequency (dependent on $C_{p a r}$ ) is not a design goal, and we have determined the layout in order to obtain the required resonance frequency and reactance slope (resulting in the parasitic capacitance $C_{p a r}$ provided in the caption of Fig. 6, where the different responses are depicted).
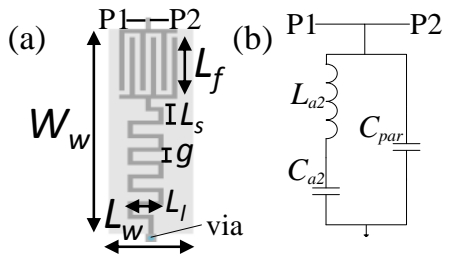

Fig. 5. Topology of the shunt-connected series resonator (a) and equivalent circuit model (b). The window etched in the ground plane is depicted in grey. The width of interdigital capacitor and narrow strip is set to $0.15 \mathrm{~mm}$. Dimensions are (in mm): $L_{f}=2.46, L_{s}=0.67, g=0.36, L_{l}=1.06, W_{w}=8.1$ and $L_{w}=2.5$. The unloaded quality factor of the LC series resonator is $Q_{u}\left(\omega_{0}\right)=69$. 

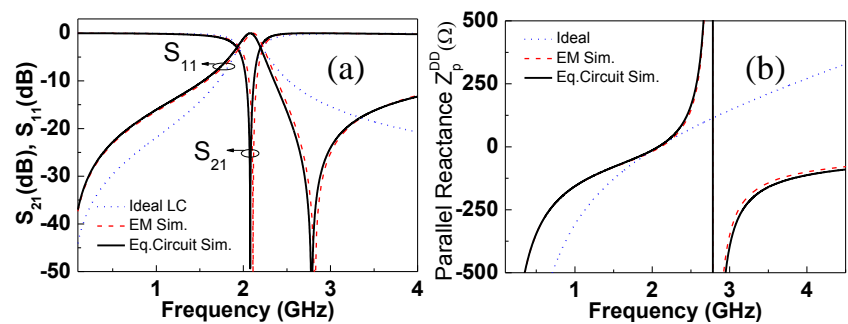

Fig. 6.S-parameters (a) and reactance (b) of the layout of Fig.5, compared to the proposed equivalent circuit and the ideal LC series resonator. The element values of the equivalent circuit are: $L_{a 2}=14.77 \mathrm{nH}, C_{a 2}=0.39 \mathrm{pF}$ and $C_{p a r}=0.50 \mathrm{pF}$. The considered substrate for the EM simulations is RogersRO3010 with dielectric constant $\varepsilon_{\mathrm{r}}=10.2$ and thickness $h=0.254 \mathrm{~mm}$.

\section{DESIGN OF THE DUAL-BAND BALANCED BANDPASS FILTER AND RESULTS}

Let us considered a 2-pole Chebyshev dual-band differential bandpass filter centered at $f_{l}=1.8 \mathrm{GHz}$ and $f_{2}=2.4 \mathrm{GHz}$, $F B W_{D B}$ equal to $7 \%$ and ripple of $0.01 \mathrm{~dB}$. From the mentioned filter specifications, the element values (in reference to Fig. 2) are: $L_{a l}=L_{b l}=1.23 \mathrm{nH}, C_{a l}=C_{b l}=4.76$ $\mathrm{pF}, L_{a 2}=L_{b 2}=14.77 \mathrm{nH}, C_{a 2}=C_{b 2}=0.39 \mathrm{pF}$, and the admittance inverters $J_{01}=J_{23}=0.02 \mathrm{~S}$, and $J_{12}=0.0209 \mathrm{~S}$. After optimization of the whole filter, the final filter layout was obtained. It is depicted in Fig. 7 together with the photograph of the fabricated prototype. Resonator layouts are those of Figs. 3 and 5, slightly modified to take into account the effects of the differential lines acting as admittance inverters. (a)

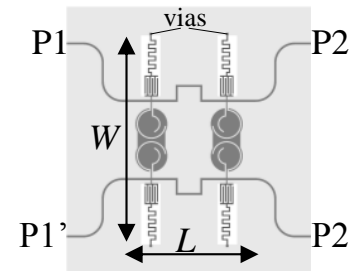

P2

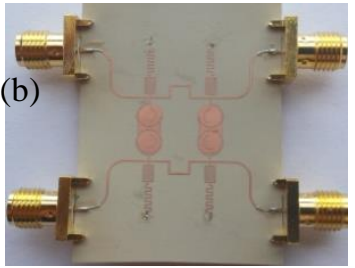

Fig. 7. Layout (a) and photograph (b) of the fabricated dual-band bandpass filter. The dimensions are: $W=27.7 \mathrm{~mm}$ and $L=17.23 \mathrm{~mm}$. The considered substrate was RogersRO3010 with dielectric constant $\varepsilon_{\mathrm{r}}=10.2$ and thickness $h$ $=0.254 \mathrm{~mm}$. The lower metal is depicted in light grey.

The agreement between the electromagnetic simulation and measurements is very good (Fig. 8). The experimental data were obtained by means of the 4-port Agilent PNA N5221A network analyzer. We also included in this figure the circuit simulation for both modes (differential and common), validating the proposed circuit models for both resonant elements, at least in the frequency range of interest. The ideal Chebyshev response is also plotted in the figure. Note that the designed filter provides more selectivity due to the presence of a transmission zero for the differential-mode at $3.8 \mathrm{GHz}$ (this avoids using a higher order, with direct impact on filter size). The measurements show a common-mode rejection ratio $(C M R R)$ of $37 \mathrm{~dB}$ and $29 \mathrm{~dB}$ at $f_{l}$ and $f_{2}$, respectively, with a rejection level higher than $19 \mathrm{~dB}$ in the whole differential pass bands. In order to appreciate the competiveness in terms of performance and size of the proposed filter, a comparison to other dual-band balanced bandpass filters with comparable $F B W$ is shown in Table I.

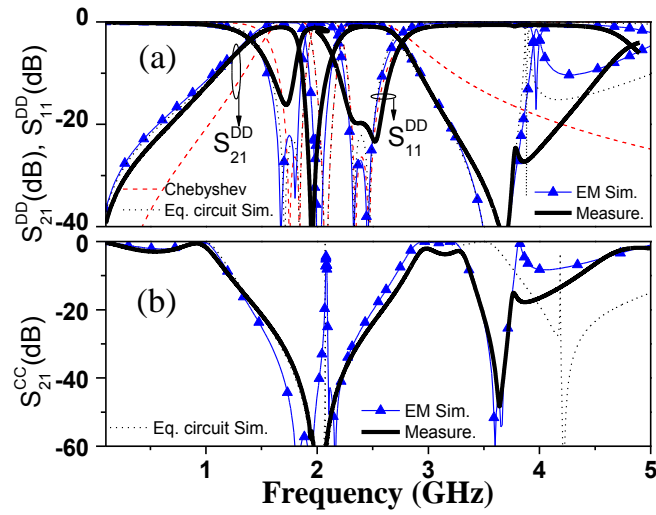

Fig. 8.Simulated and measured differential- (a) and common-mode (b) $S$ parameters. The differential- and common-mode circuit simulations and the ideal dual-band Chebyshev response are also included.

TABLE I

COMPARISON OF VARIOUS DUAL-BAND DIFFERENTIAL-MODE BANDPASS FILTERS

\begin{tabular}{c|c|c|c|c} 
Ref. & $\begin{array}{c}\boldsymbol{F B} \boldsymbol{W}_{1} / \boldsymbol{F B} \boldsymbol{W}_{2}( \\
\mathbf{\%})\end{array}$ & $\begin{array}{c}\boldsymbol{C M R R} @ \\
f_{I} / \boldsymbol{f}_{2}(\mathbf{d B})\end{array}$ & $\begin{array}{c}\text { Lumped } \\
\text { Elements }\end{array}$ & $\begin{array}{c}\text { Effective } \\
\text { area }\end{array}$ \\
\hline$[2]$ & $12.5 / 25$ & $15 / 22$ & No & $0.06 \lambda_{g}{ }^{2}$ \\
\hline$[3]$ & $15.2 / 6.6$ & $53 / 43$ & Yes & $0.13 \lambda_{g}{ }^{2}$ \\
\hline$[5]$ & $7.5 / 6.5$ & $28 / 30$ & No & $0.12 \lambda_{g}{ }^{2}$ \\
\hline Thiswork & $\mathbf{7 / 7}$ & $\mathbf{3 7 / 2 9}$ & No & $0.10 \lambda_{g}{ }^{2}$ \\
\multicolumn{5}{|c}{$\lambda_{g}$ is the guided wavelength at $f_{l}$}
\end{tabular}

\section{CONCLUSIONS}

In conclusion, it has been demonstrated that compact dualband balanced bandpass filters with good differential-mode selectivity at the upper band and intrinsic common-mode rejection can be implemented by combining OCSRRs and resonators implemented by means of meandered inductors and interdigital capacitors. The proposed topology provides small size, good differential-mode response, and efficient commonmode rejection in the differential passbands. If the application requires further common-mode rejection bandwidth, cascaded common-mode filters may be added.

\section{REFERENCES}

[1] J-E. Lim, M-H. Nam, H-O. Choi, and J-H. Lee, "Two-port balanced dual-band bandpass filter based on stepped impedance resonators," Proc. Asia-Pacific Microw. Conf., Yokohama (Japan), 7-10 Dec. 2010.

[2] X. Chen, G. Han, R. Ma, J. Gao, and W. Zhang, "Design of balanced dual-band bandpass filter with self-feedback structure", ETRI Journal, vol. 31, no. 3, pp. 475-477, Aug. 2009.

[3] J. Shi, and Q. Xue, "Dual-band and wide-stopband single-band balanced bandpass filters with high selectivity and common-mode suppression", IEEE Trans. Microw. Theory Techn., vol. 58, pp. 2204-2212, Aug. 2010.

[4] Y.-H. Cho, I.-S. Jeon, X.-G. Wang, S.-W. Yun, and D.-C. Park, "Balanced dual-band bandpass filter using microstrip resonators loaded with lumped-elements", Proc. Asia-Pacific Microw. Conf., Melbourne (Australia), 5-8 Dec. 2011.

[5] Y.-H. Cho, and S.-W. Yun, "Design of balanced dual-band bandpass filters using asymmetrical coupled lines", IEEE Trans. Microw. Theory Techn., vol. 61, pp. 2814-2820, Aug. 2013.

[6] G. L. Matthaei, and L. Young, E.M.T. Jones, Microwave filters, impedance matching and coupling structures, Artech House, 1985.

[7] X. Guan, Z. Ma, P. Cai, Y. Kobayashi, T. Anada and G. Hagiwara, "Synthesis of dual-band bandpass filters using successive frequency transformations and circuit conversions", IEEE Microw. Wireless Comp. Lett., vol. 16, pp. 110-112, Mar. 2006.

[8] P. Velez, M. Duran-Sindreu, J. Naqui, J. Bonache, and F. Martín, "Common-mode suppressed differential bandpass filter based on open complementary split ring resonators (OCSRRs) fabricated in microstrip technology without ground plane etching", Microw. Opt. Technol. Lett., vol. 56, pp. 910-916, Apr. 2014. 